\section{Ciencia y persuasión social en la medicalización de la infancia en España, siglos XIX-XX*}

\section{Science and social} persuasion in the medicalization of childhood in 19th-and 20th-century Spain

\section{Esteban Rodríguez Ocaña}

Departamento de Anatomía Patológica e Historia de la Ciencia

Facultad de Medicina, Universidad de Granada 18071, Granada, España erodrig@ugr.es

\section{Enrique Perdiguero}

Departamento de Salud Pública, Historia de la Ciencia y Ginecología

Universidad Miguel Hernández

Crta. Alicante-Valencia, s/n

03550 Sant Joan d'Alacant, Alicante, España quique@umh.es
RODRÍGUEZ OCAÑA, E.; PERDIGUERO, E.: Ciencia y persuasión social en la medicalización de la infancia en España, siglos XIX-XX.

História, Ciências, Saúde - Manguinhos, v. 13, n. 2, p. 303-24, abr.-jun. 2006.

Este trabajo indaga la conversión en rutinaria de la visita del médico de niños en España. Estudia la sustitución de modelos de atención al embarazo, parto y crianza, tradicionalmente opacos para varones y objeto de cuidados mediante agentes populares, por otros accesibles a la medicina académica. A partir de la situación existente a nivel popular en el último tercio del siglo XIX, exploramos la repercusión de campañas de divulgación científica - entendidas como crítica sin cuartel a lo que se construyó como cultura popular - y la proliferación de dispositivos asistenciales gratuitos. La oferta de vigilancia sobre la alimentación de lactantes permitió familiarizar a las madres con la asistencia facultativa en la enfermedad, hasta que, en la segunda mitad del siglo XX, la Pediatría se centró preferentemente en las patologías y la Puericultura dejó de ser especialidad médica para convertirse en identidad profesional subalterna.

PALABRAS CLAVE: puericultura; medicina popular; popularización; España; siglos XIX y XX.

RODRÍGUEZ OCAÑA, E.; PERDIGUERO, E.: Science and social persuasion in the medicalization of childhood in 19th- and 20th-century Spain.

História, Ciências, Saúde - Manguinhos, v. 13, n. 2, p. 303-24, Apr.-June 2006.

The article explores how childhood visits to doctors first became routine in Spain. The introduction of new models of prenatal care, childbirth, and childrearing required the extension of academic medicine into a terrain traditionally occupied by practitioners of popular medicine. Focusing on the status quo for most of the population in the final third of the nineteenth century, the study examines the repercussion of the era's scientific outreach campaigns (expressions of harsh criticism of what popular culture had constructed) and the spread of free health assistance. In particular, it highlights how attention to the nutritional needs of nursing mothers helped these women gain familiarity with the medical assistance available in the case of illness - so much so that by the second half of the twentieth century, the issues of health education and promotion had been relegated to a secondary plane within the medical profession.

KEYWORDS: childrearing; popular medicine; popularization; Spain; nineteenth and twentieth centuries. 
* Trabajo realizado con financiación del proyecto BHA20012979-C05 de la DGIMinisterio de

Educación y Ciencia (España).
$\mathrm{E}$ 1 Anuario sanitario de España de 1909, una publicación no oficial, que respondía a intereses comerciales y propagandísticos y listaba, con nombre y dirección, casi 14.000 profesionales, revela una escasísima presencia de especialistas en medicina de la infancia: sólo aparecen reseñados como tal 10 médicos en toda España, junto con otros 12 médicos de partos, ninguno en las dos grandes capitales; ni siquiera aparecen con denominación especializada los catedráticos de Pediatría existentes en aquella fecha, entre quienes al menos los de Madrid, Barcelona, Valencia y Zaragoza fueron prolíficos autores en este campo. En 1946, la misma fuente y sólo en capitales de provincia señala la especialización materno-infantil de un $12,7 \%$ del total de los profesionales: unos 800 pediatras y puericultores y 727 tocólogos.

¿Qué sentido encierran estas cifras?

La especialización profesional se inició como una actividad subordinada a un trabajo generalista; por sí misma no garantizaría los ingresos de un profesional. De ahí la poca utilidad de emplearla como reclamo a principios de siglo, incluso por parte de aquellos más directamente implicados en su desarrollo. Cuarenta años después, sin embargo, la denominación como especialistas identifica claramente una gestión profesional autónoma y suficiente; existe un reconocimiento, una visibilidad social de la figura del especialista. El especialista tiene un público.

El proceso que generó la especialidad de medicina de niños, o Pediatría, tuvo distintos componentes. Uno, que no vamos a tratar, es el de sus contenidos técnicos, el de la conversión del cuerpo infantil en objeto de estudio y de práctica científica. Por otra parte, el nacimiento y desarrollo de la especialidad estuvo vinculado con una determinada concepción social de la infancia - esto es, con la puesta en valor de esa etapa de la vida humana a escala poblacional, en base a sus beneficios para la familia, la nación y el estado (Ballester y Balaguer, 1995). Ese desarrollo exigió sustituir los modelos de atención al embarazo, parto y crianza, tanto en sus contenidos como en sus agentes, merced a lo cual se extendió el ámbito jurisdiccional de la medicina universitaria a procesos tradicionalmente opacos para los varones y gestionados por agentes populares o simplemente por las redes sociales primarias. Hubo que generar un público para la especialidad así como el suministro de profesionales para recibirlo y organizar las instituciones (dispositivos de beneficencia, seguro de maternidad y seguro de enfermedad).

Los autores de este trabajo han puesto en común su familiaridad con las fuentes temáticas pertinentes, que hasta ahora habían manejado por separado y cubriendo aspectos complementarios (Rodríguez Ocaña, Ortiz y García-Duarte, 1985; Perdiguero, 1993 y 1995; Perdiguero y Bernabeu, 1995, 1997 y 1999; Rodríguez Ocaña, 1996 y 1999). Estamos convencidos de que este acercamiento común 
facilitará la mejor comprensión de la complejidad del proceso de formación de la especialidad de Pediatría en España entre los siglos XIX y XX.

\section{Algunas razones y un punto de partida}

Como hemos advertido, el convertir en rutinaria la visita del médico exigió sustituir las prácticas tradicionales del cuidado de la infancia introduciendo en la cultura popular, como dominante, una delimitación científica de las entidades 'niño' y 'madre' (Apple, 1980 y 1995). Este objetivo se situó en un plano prominente en España en el momento del cambio de siglo debido al ascenso de la clase médica profesional, en razón del grado de desarrollo burgués, a la prioridad del proyecto regeneracionista y a la conciencia extendida entre las elites de la mortalidad infantil como problema social.

Para conseguirlo se combinaron: a) una campaña de crítica implacable a los contenidos de lo que se construyó como 'cultura médica popular'; b) la oferta de dirección científica y de vigilancia profesional del embarazo, parto y crianza, a través de dispositivos gratuitos (dispensarios municipales y obras benéficas privadas); c) la posibilidad de alimentar a los niños mediante biberón, con leches garantizadas desde el punto de vista sanitario, a precios asequibles; $y, d)$ el libre acceso al médico en caso de enfermedad a través de esas mismas instituciones benéficas. Además, en todo momento la práctica institucional fue, por sí misma, educativa, al sustituir viejas por nuevas rutinas (por ejemplo, si no se realizaba el pesado periódico de los niños se perdían los beneficios de leche barata, ropa gratuita u otros), a la vez que era también expresamente formadora mediante cursos, conferencias, escuelas de maternologías, etc.

${ }^{1}$ Utilizamos aquí 'educación sanitaria', no con el perfil de actividad técnica que tiene en la actualidad, sino como suministro a la población de conocimientos médicos actualizados, es decir, algo similar a la popularización o divulgación sanitaria.
La educación sanitaria ${ }^{1}$ (incluyendo la aceptación del trabajo experto de médicos pediatras) aparecía como la vía regia de la defensa de la nación en momentos en que el leit motiv político se cifraba en la consigna de 'regeneración', tras la derrota por Estados Unidos y la pérdida de las últimas posesiones de ultramar, y aparecía un reformismo social para hacer frente a los nuevos problemas derivados de las reivindicaciones obreras que incluía la denominada 'medicina social' (Rodríguez Ocaña, 1987 y 2006). La medicina se asignaba la tarea de 'pacificar' a la gran masa de la sociedad española. Sólo los médicos tendrían el derecho y el deber, fundado en la naturaleza de sus conocimientos profesionales, de guiar la vida de la colectividad; no ya gobernar, sino señalar "la única senda que conduce a la prosperidad" que coincidiría con la buena salud del pueblo (Tolosa, 1903, p. 10-1). Una posición como consejeros áulicos que ya venía siendo ejercida por algunas instituciones médicas como la Real Academia de Medicina de Madrid o la Sociedad Española de Higiene, controladas por miembros de la oligarquía 
política de la Restauración pertenecientes a ambos partidos dinásticos, en cuestiones como los planes de ensanche y reforma urbanística de las ciudades o el saneamiento urbano (Rodríguez Ocaña, 2001). Pero si en estos terrenos, el protagonismo de los médicos se subordinó al de arquitectos e ingenieros o, en el terreno de la previsión social, al de abogados, economistas y actuarios, en función de la diversificación profesional burguesa, en el campo de la asistencia a la infancia sólo tuvo que compartir competencias con maestras y pedagogos, y siempre desde una posición dominante.

En cuanto al concepto de 'cultura médica popular' no fue un préstamo tomado de las ciencias sociales, sino que fue puesto a punto por los propios médicos con el fin de diferenciar su práctica de la ejercida por la población, legitimando así su manera de entender la salud y la enfermedad (Martínez y Comelles, 1994). Frente a la universalidad y eficacia de 'la medicina de los médicos' se encontraba el localismo y la ineficacia de la medicina popular. En esta tarea los médicos no estuvieron solos. Los folcloristas españoles (algunos, médicos de profesión) de finales del siglo XIX, en Andalucía, Extremadura, Asturias o Cataluña, llevaron a cabo investigaciones, casi siempre a través de cuestionarios, que compusieron la imagen de una medicina popular ilógica y estable en su desvarío, gran obstáculo para la penetración de la medicina científica (Perdiguero y Bernabeu, 2003).

El proceso de creación de la especialidad de 'medicina de niños' se sustentó sobre unos círculos institucionalizados de aprendizaje y práctica profesional (hospitales, cátedras, dispensarios), unos circuitos de publicación (revistas especializadas) y unas medidas de defensa e intercambio profesional, como las sociedades de especialistas. Dicho proceso ocurrió de forma más precoz en Francia desde donde los saberes médicos sobre niños se difundieron hacia España tanto en forma de traducciones de las principales monografías como de reseñas en las publicaciones periódicas médicas. En los decenios iniciales del siglo XX hay que advertir una no menos importante influencia germana (Seidler, 1974; La Berge, 1991; Klaus, 1993).

En las facultades, estos saberes se encontraban inicialmente unidos a la Obstetricia y Ginecología, como 'Clínica de partos y enfermedades de mujeres y niños' (Plan de estudios de 1845) u 'Obstetricia y Patología de la mujer y los niños' (plan de 1857), hasta que a partir de 1886 aparecieron otras como 'Enfermedades de los niños' y 'Pediatría' (desde 1928). En 1876, se fundó el primer centro especializado, Hospital del Niño Jesús, en Madrid, promovido por una asociación caritativa que pretendió, sin conseguirlo, la instalación de uno en cada capital de provincia. El director más conspicuo de los primeros tiempos del hospital madrileño fue Mariano Benavente (1818-1885), médico de los niños de la aristocracia cortesana y primer especialista español de Pediatría, según 
Granjel (1965), cuya clientela heredó Manuel Tolosa Latour (18571919). Este promovió otra asociación para la construcción de sanatorios marinos, según los acuerdos del Primer Congreso Internacional de Protección a la Infancia (Bruselas, 1883), que se concretó en 1897 en la instalación de un centro en Chipiona, provincia de Cádiz. En otros hospitales benéficos, en particular los adscritos a las Facultades de Medicina, se dotaron salas de clínicas para niños desde mediados de los años ochenta del siglo XIX (figura 1). Por su parte, las consultas ambulatorias se abrían en relación con las necesidades docentes, aunque con notable irregularidad de funcionamiento y adscripción. Con recursos privados, al menos otros cinco hospitales y numerosos dispensarios o consultorios para niños enfermos se abrieron antes de 1910 en distintas ciudades. Igualmente, desde la beneficencia municipal algunas grandes ciudades crearon servicios especializados para niños enfermos. En Madrid, por ejemplo, en 1892 se instaló una primera 'consulta para niños enfermos y pobres' en la Casa de Socorro del distrito centro y en 1893 se instaló otra 'consulta para niños' en la nueva Casa de Socorro del distrito de palacio, ampliada en 1913 para crear la Institución Municipal de Puericultura.

Por lo que respecta a la conciencia de alarma social acerca de la mortalidad infantil, se puede advertir la proliferación de estudios demográfico-sanitarios apoyados en la explotación de los registros civiles (Aguirre, 1885, sobre Madrid; Borobio, 1893, sobre Zaragoza o Comenge, 1899, sobre Barcelona, entre muchos otros; cf. Gómez Redondo, 1992). Prácticamente hasta los trabajos de Marcelino Pascua (1934), más preocupados por el rigor técnico, los textos de este tipo manejaron una 'retórica de la catástrofe', encaminados a

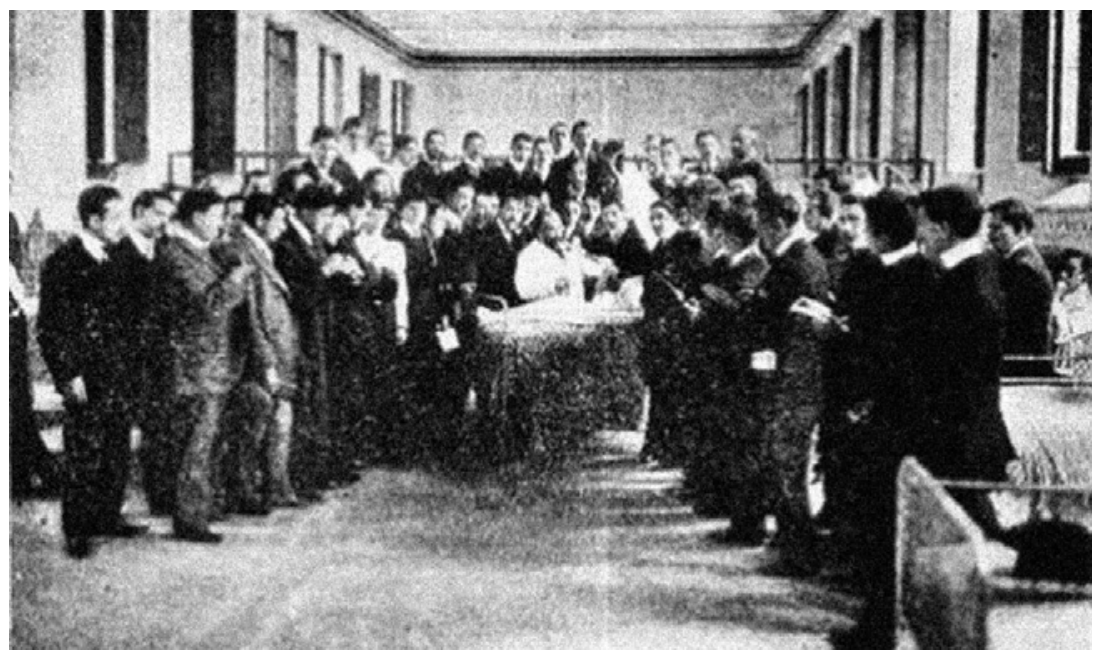

Figura 1 - Docencia pediátrica de Andrés Martínez Vargas en el Hospital Clínico de Barcelona. Fuente: Martínez Vargas, A. Tratado de Pediatría, Barcelona, 1915. 
movilizar la opinión pública, muchas veces en apoyo de intervenciones médico-sociales concretas como en el caso ejemplar del Informe acerca de la mortalidad infantil de Madrid, sus principales causas y medios de combatirla, presentado por Rafael Ulecia en 1903 a la Junta Municipal de Sanidad.

\section{Pautas tradicionales en la atención materno-infantil}

Para conocer cuáles eran las pautas tradicionales en la atención materno-infantil en la segunda mitad del siglo XIX, además del estudio de ciertas fuentes materiales, los principales informadores son, precisamente, los autores implicados en la campaña que estamos comentando, quienes describían los usos y costumbres populares para criticarlos, si bien dado su objetivo hemos de aplicar todas las cautelas a la información que nos han transmitido. Algunas fuentes son especialmente ricas, como es el caso de Madre e hijo. Doctrina científica y errores populares en obstetricia y ginecología (Madrid, 1898), cuyo autor, Enrique Salcedo Ginestal, cursó a todos los sanitarios titulados de los pueblos de España y a muchos de ciudades, la petición de que le transmitieran toda clase de "errores, supersticiones, refranes y frases" populares en relación con el tema de su estudio. A diferencia de los folcloristas, en trabajos como el de Salcedo, rara vez se trata de mostrar lo positivo de las prácticas populares, sino exponer su "lado ridículo ... y aun criminal". Había que combatir sin piedad "la mentira, el error, la mala fe" (Salcedo, 1898, p. XII), omnipresentes en el mundo popular.

El ya mencionado Mariano Benavente recordaba de sus primeros años de ejercicio, alrededor de 1855, en Villarejo de Salvanés, una pequeña población castellana, que las enfermedades infantiles más frecuentes del lugar eran "la baba, el asiento colado y el mal de ojo" (Benavente, 1883a). Este último padecimiento recibió especial atención a partir del libro de Rafael Salillas (1905) elaborado a partir de la Información promovida por la Sección de Ciencias Morales y Políticas del Ateneo de Madrid, en el campo de las costumbres populares y en los tres hechos más característicos de la vida: el nacimiento, el matrimonio y la muerte, llevada a cabo en los inicios del siglo XX (Perdiguero, 2004, p. 140-2).

La baba y el asiento eran también reconocidos en medios populares urbanos como los principales males de la infancia. El asiento (Bernabeu, 1995 y 2002a, p. 153-8), por su parte, parece ser el diagnóstico popular que se daba a síntomas como la indigestión y el estreñimiento, e inducía a intervenir para evitar la acumulación de sustancias nocivas, mediante purgantes, fricciones y otras manipulaciones. Un médico de la beneficencia municipal de Madrid señalaba que 'levantar el asiento' era el mayor título que adornaba a las curanderas o saludadoras de común recurso en medios popu- 
lares (Aguirre, 1885, p. 168-9). Los 'amasamientos' de la terapéutica popular fueron asimilados por la medicina, como operaciones empíricas beneficiosas, acompañándolas de explicaciones fisiopatológicas e integrándolas en técnicas expertas (Salcedo, 1898, p. 661).

La enfermedad de la baba se caracterizaría por la emisión de flujos o humores nocivos e incluía todas las inflamaciones / supuraciones y diarreas. En los momentos de la dentición, baba y diarrea eran sinónimas y su presencia era tenida por saludable, hasta el punto que, de no producirse la segunda, se intentaba provocarla por medios artificiales, mediante jarabes. Desde la medicina se pretendió asimilar estas nociones bajo el diagnóstico de 'accidentes de la dentición' y enfatizar el peligro de estimular la diarrea o menospreciar su presencia; pero, la concepción de la diarrea como fenómeno saludable se mantuvo vigente para muchas madres hasta bien entrado el siglo XX (Huergo, 1885, p. 87-8; Lozano, 1883, p. 154-7; Arteche, 1928, p. 62).

¿Podemos caracterizar la relación entre la cultura científica y la cultura popular en términos de absoluta oposición? Tal parece, pero hubo algunas simbiosis. El análisis de la etiqueta diagnóstica 'dentición' muestra la existencia de un verdadero proceso de aculturación en la medida en que los contenidos popularmente aceptados (y científicamente rechazados) a finales del siglo XIX procedían de lo que era doctrina médica un siglo antes (Perdiguero y Bernabeu, 1995). La colonización del imaginario popular por conceptos científicos fue advertida en su momento, subrayándose la disparidad en la evolución de ambos medios como la causa de la falta de sincronía: “la profesión médica origina el nacimiento de ... términos vagos, de muy amplio significado ... en la práctica no carecen de inconvenientes ... uno de ellos es que el vulgo acabe por incorporarse estos conceptos generales" (Juarros, 1919, p. 70-3). Al mismo tiempo, la evolución del pensamiento médico en torno a la alimentación infantil (como, por ejemplo, la figura de las amas de leche, que examinamos más adelante) muestra la influencia que las prácticas populares, sancionadas por tradiciones y experiencias seculares, ejercieron sobre los contenidos de las propias propuestas científicas, en absoluto ajenas a su contexto sociocultural.

Lo que se vivía a finales del siglo XIX era un episodio de cambios rápidos en las concepciones médicas que se enfrentaban con la lentitud de adaptación de conceptos y prácticas tradicionales, contra las que tenían que desarrollarse, en particular asentando la función profesional médica. Un comentario esclarecedor de 1891 refiere cómo "[e]n no pocas ocasiones paga el 'mal de ojo', a las 'espaldas' de los médicos, los vidrios rotos de la mala dirección en la lactancia; puesto que los niños adquieren enfermedades difíciles o imposibles de curar" (Valera, 1891, p. 29). La conclusión obvia, 
tanto en 1891 como en 1925, es que hacía falta más educación higiénica, a cuyo servicio se instauró una 'medicina preventiva o profilaxia higiénica' (Canoura, 1925, p. 12) que en el campo de la crianza infantil se llamó Puericultura.

\section{La Puericultura como vía de familiarización con la medicina}

El objeto de la Puericultura era extender las normas científicas de cuidado de la infancia, en particular las referidas al régimen alimenticio durante la lactancia. Fue la expresión profesional de la campaña de prevención de la mortalidad infantil que comenzó bajo dirección y vinculación exclusivamente médica para pasar, más adelante, a finales de los años de la década de 1960, a convertirse en un nicho profesional subalterno en la escala sanitaria (auxiliares y matronas). Sin embargo, había sido la primera especialidad médica reglada de España, dotada con un centro de formación (la Escuela Nacional de Puericultura, legislada en 1923 y hecha realidad a partir de 1926), cuyos titulados fueron, o bien oficiales sanitarios que completaban sus destrezas en el marco exigido por la medicina social de la época (entre 1926 y 1960, aproximadamente), o bien fueron médicos de niños, pediatras, que igualmente completaban su formación con los aspectos higiénicos, preventivos y de promoción de la salud de la infancia.

Originalmente, la Puericultura y los puericultores promocionaron la alimentación maternal. De los tres modos genéricos de proporcionar alimento al neonato - lactancia materna, lactancia por nodriza y lactancia artificial (especialmente mediante biberón) - los médicos se declararon unánimemente defensores de la primera, rígidos censores de la segunda y absolutos enemigos de la última. Pero los resultados fueron contradictorios, puesto que la campaña puericultora condujo a un aumento del uso del biberón. Y es que el compromiso técnico de la Puericultura se reforzó en el terreno de la lactancia artificial donde más claramente accesibles eran los instrumentos y las reglas externas a los cuerpos de las mujeres, a la vez que permitía atender las necesidades que generaba la irrebatible salida de la mujer del hogar con los menos perjuicios para la salud pública (Wolf, 2001).

Los centros puericultores nacieron de la combinación de la educación higiénica y los socorros benéficos (el suministro gratuito o semigratuito de leche, así como otros alimentos para los lactantes y, en algún caso, para las madres, además de otras medidas benéficas tradicionales como ropa, canastillas, incluso dinero). Tuvo prioridad la tecnología de la alimentación artificial, que servía de reclamo para atraer una clientela popular (figura 2). En España existieron gotas de leche a partir de 1902 y consultorios para lactantes desde 1904, casi todos incluyendo un servicio de 
esterilización y reparto de leche, a semejanza del primero organizado en Madrid por Rafael Ulecia (1850-1912) (Rodríguez Ocaña, Ortiz y García-Duarte, 1985) (figura 3). En el momento de máxima intensidad de las actuaciones públicas puericultoras, en torno a 1956, no se alcanzaron los 500 centros en toda España, lo que quedaba muy lejos de lo que fue habitual en los países europeos de los que se copiaba dicha práctica; en Francia existían en 1933 más de 4.500 o en Bélgica funcionaban no menos de 900 consultorios de lactantes de titularidad pública en 1924 (Rollet-Echalier, 1990, p. 387 y 392).

La vertiente de educación popular se plasmó de manera más explícita en la creación de las Escuelas de Maternología, destinadas a proporcionar formación puericultora a otras mujeres, jóvenes escolares y madres. Durante la República, cada dispensario tenía la obligación de realizar una conferencia semanal durante seis meses cada año, para las madres atendidas, además de realizar cursos en las escuelas municipales, obligatorios para las alumnas. En 1930 se calculaba, en Madrid, que unas 18.000 madres y alrededor de 6.000 escolares habían atendido las conferencias desde su inicio en 1918 y 1921, respectivamente. Como se muestra en otro artículo de este mismo número también se utilizaron otros medios de persuasión como carteles, emisiones de radio y películas. Esta enseñanza especializada completaba una formación genérica, impartida en las escuelas desde mediados del siglo XIX, particularmente dirigida a las niñas, y que buscaba la sustentación de una 'moral de la

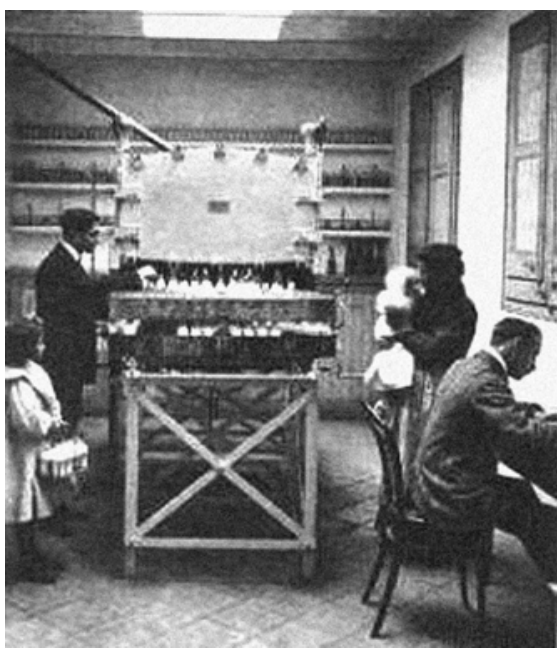

Figura 2 - Sala de máquinas de la Casa de Lactancia de Barcelona, c. 1916.

Fuente: Arrizabalaga, J.; Martínez Vidal, A.; Pardo Tomás, J. La salut en la història d'Europa, Barcelona, Residencia d’Investigadors-CSIC,1998 [portada].

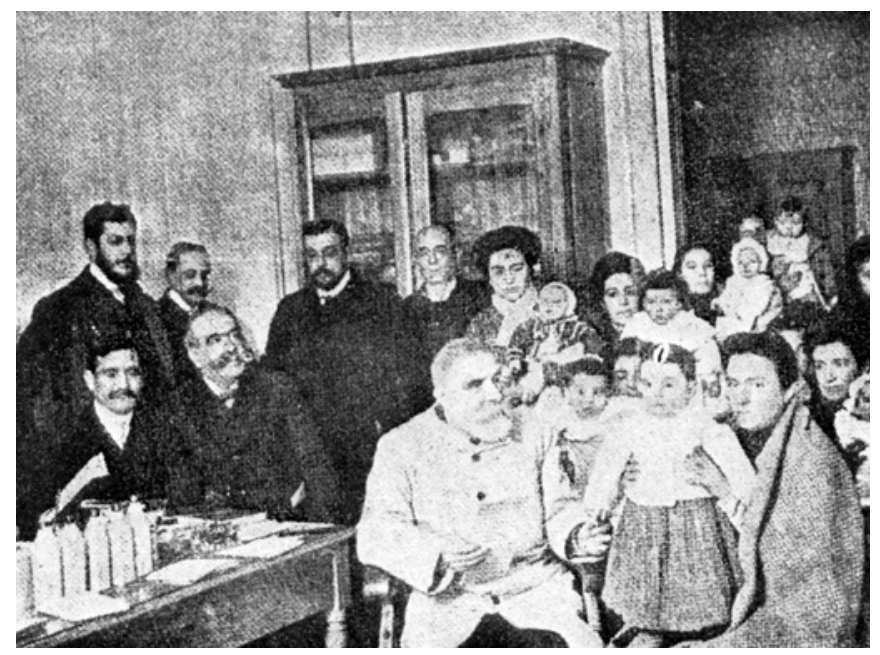

Figura 3 - Consulta del Dr. Ulecia en el Primer Consultorio de Niños de Pecho de Madrid, 1904. Fuente: Libro conmemorativo del cincuentenario (bodas de oro) de la institución Primer Consultorio de Niños de Pecho, en Madrid (Gota de Leche), 22-1-1904, 22-1-1954, Madrid, 1954. p. 60. 
domesticidad'; enseñanza que sería reforzada bajo el franquismo con el explícito nombre de 'enseñanzas del hogar', vigente hasta 1970. Recientemente se ha realizado un estudio pormenorizado de sus contenidos y fines, empleando como material un número importante de los textos usados en su docencia (de Haro, 1999), si bien, como muestra la tabla 1, los aspectos de la crianza quedaban difuminados en el marco de las preocupaciones higiénicas generales acerca de la vivienda, el aseo personal o el vestido.

Tabla I - Principales asuntos tratados en los textos destinados a las enseñanzas domésticas en España (1857-1970)

\begin{tabular}{lccc}
\hline & $\begin{array}{c}1857-1900 \\
(44 \text { de } 94 \text { textos*) }\end{array}$ & $\begin{array}{c}1901-1939 \\
(39 \text { de } 58 \text { textos*) }\end{array}$ & $\begin{array}{c}1940-1969 \\
(100 \text { de 275 textos*) }\end{array}$ \\
\hline $\begin{array}{l}\text { Condiciones de } \\
\text { la vivienda }\end{array}$ & $61 \%$ & $52 \%$ & $48 \%$ \\
\hline Vestido & $70 \%$ & $44 \%$ & $37 \%$ \\
\hline Aseo corporal & $63 \%$ & $50 \%$ & $49 \%$ \\
\hline Régimen alimenticio & $54 \%$ & $44 \%$ & $39 \%$ \\
\hline Cuidados a personas & $27 \%$ & $29 \%$ & $19 \%$ \\
\hline
\end{tabular}

* NN textos analizados del total de YY autorizados en cada periodo.

Fuente: Sintetizado a partir de los datos suministrados por Isabel M $\mathrm{M}^{\mathrm{a}}$ de Haro Oriola. La educación de las mujeres como promotoras de salud en la España contemporánea. Cambios y pervivencias (Tesis doctoral, Universidad de Granada, 1999; apartados 3.1.1, 3.1 .2 y 3.1.3).

En el último tercio del ochocientos apareció una notable actividad editorial sobre 'consejos a las madres', monografías con clara intención popularizadora que perduró hasta mediados de la década de 1960. Así, por ejemplo, los Consejos prácticos sobre la higiene de la primera infancia de Vidal Solares, con diez ediciones entre 1882 y 1915 , más un número indeterminado, con seguridad más de quince, de los mismos en formato reducido (titulado Instrucción dialogada [o preceptos] sobre puericultura e higiene de la primera infancia, a veces precedido por consejos a las madres) entre 1886 y 1916; también el caso del Arte de criar a los niños de Rafael Ulecia, con cinco ediciones entre 1904 y 1924, o el Catecismo de puericultura de Juan Bosch Marín, catorce ediciones entre 1933 y 1966. Bajo la forma sintética de 'cartillas sanitarias de la primera infancia' o 'de puericultura', se multiplicaron, asociadas a las distintas instituciones locales benéficas u oficiales locales enfocadas hacia la asistencia materno-filial y aparecieron con enorme frecuencia en la prensa política dentro de las secciones que se dedicaban a las noticias relacionadas con la medicina (Perdiguero, 1993). Está claro que su objetivo era contribuir a la transformación en el seno de las familias de las pautas tradicionales de cuidados materno-infantiles, sistemáticamente denigradas como perjudiciales, por otras científicas dependientes del consejo médico experto. 
La Dirección General de Sanidad creó el Servicio de Higiene Infantil en 1932, con el objeto de llevar la atención puericultora al mundo rural a través de los centros de higiene comarcales y locales. Para su dotación se contaba con la existencia de la Escuela Nacional de Puericultura que, entre 1927 y 1963, creó hasta 16 centros de ámbito regional o provincial asociados. Esta titulación resultaba indispensable para cubrir las plantillas del servicio, que, después de la guerra, continuó las líneas programáticas iniciadas en la República, confirmadas por la ley de sanidad materno-infantil de 1941, de manera que en 1956 se alcanzaba el máximo de dotaciones en centros comarcales (unos 107). Para su actuación, la sanidad pública contó con el auxilio inestimable de dos organizaciones, brazos sociales del Movimiento Nacional (partido único franquista): Auxilio Social y Sección Femenina.

La Obra Nacional de Auxilio Social (López, 1967; Orduña, 1996; Carasa, 1997) se desarrolló formalmente desde 1937. Tuvo competencias en la gestión de gotas de leche, dispensarios de maternología, maternidades, comedores para madres gestantes y lactantes, hogares para huérfanos y abandonados, guarderías, jardines maternales y otros centros. Fue una de las vías por las que, a partir de septiembre de 1954, se distribuyó la contribución de la National Catholic Welfare Conference de Estados Unidos - la ayuda social americana -, básicamente compuesta de productos alimenticios, para cuyo reparto se crearon unas juntas asistenciales por provincias y que alcanzó a unos cuatro millones de beneficiarios menores de edad.

La Sección Femenina no se limitó a una actividad institucional, sino que participó de manera decisiva en las tareas de divulgación (Sánchez, 1990, p. 34 y 40-3; Gallego, 1983, p. 124-5; Mata, 1994; Bernabeu, 2002b). Formó un cuerpo de voluntarias que, luego de un curso de formación básica, eran enviadas a los pueblos en misiones de inspección, vacunación, reparto de medicamentos y de ropa y enseñanza de rudimentos higiénicos. Gracias a su intervención, la Dirección General de Sanidad desarrolló una campaña contra los trastornos alimenticios, realizando entre 1941 y 1946 más de millón y medio de visitas y atendiendo a unos 180.000 niños. A partir de 1947 organizó cátedras ambulantes de Puericultura que conectaron con muchos miles de personas en el medio campesino.

El Seguro de Enfermedad, iniciado en 1944, heredó de la República su preparación técnica y la dotación del Seguro de Maternidad que había comenzado a funcionar en octubre de 1931. La Obra Maternal e Infantil del Seguro de Maternidad intervino en la atención higiénica y médica posparto, para lo que disponía de 12 dispensarios propios y 60 concertados en 1935, que pasaron a ser 250 consultorios propios y 9 residencias en 1947, todo lo cual se integró en el Seguro Obligatorio de Enfermedad. Entre 1956 y 1964, 
el Seguro de Enfermedad gestionó 8 residencias maternales (maternidades), 185 consultorios de pediatría-puericultura y 99 de maternología (Bernabeu-Mestre y Perdiguero-Gil, 2001) (figura 4).

La puesta en marcha de medidas de previsión social en relación con el parto implicaba el acceso de la medicina a territorios escasamente visitados hasta entonces. En Zaragoza, se estimó que con anterioridad a la entrada en vigor del Seguro de Maternidad, más del $90 \%$ de los partos eran atendidos por 'una comadrona o una aficionada' (Gómez Salvo y Camón Gironza, 1936, p. 6). El Seguro se configuró sobre la asistencia a los partos normales a domicilio, encomendada autónomamente a matronas, mientras que el Reglamento de Servicios Sanitarios del Seguro Obligatorio de Enfermedad de enero de 1948 las convirtió en auxiliares del médico en todos los casos y estimuló el parto sanatorial, al ofrecer hasta 8 días de ingreso gratuito en institución cerrada para partos normales. La hospitalización tocológica se vio favorecida por la dotación de clínicas propias del Seguro, de nueva construcción, distintas de los tradicionales centros benéficos destinados a pobres; también, por el empleo de la indicación llamada 'distocia social' - ingreso por malas condiciones de habitabilidad o de falta de cuidados en el hogar propio (aplicada en el 61,3\% de los registrados entre 1947 y 1949). De esta forma, la hospitalización y la participación del médico en caso de parto, infrecuentes antes de la guerra civil, pasaron a ser sucesos familiares e imprescindibles. El plan de construcción de Residencias Maternales (en realidad, hospitales materno-infantiles), que se inició en la década de 1960, perseguía explícitamente "conseguir la

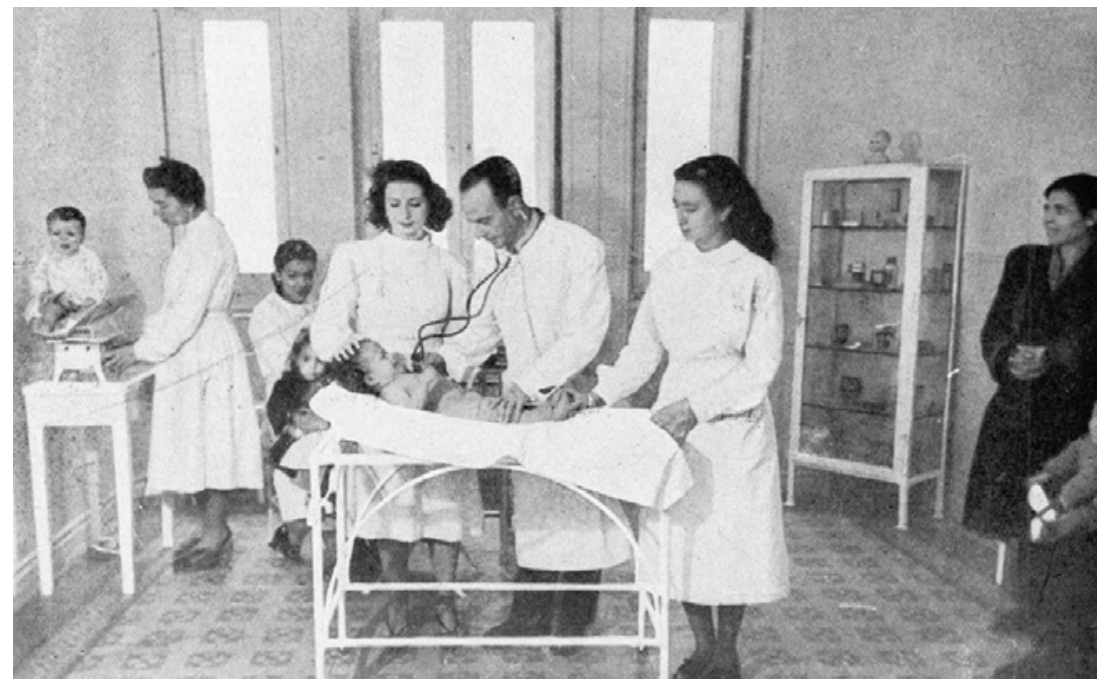

Figura 4 - Consulta médica en el Primer Consultorio de Niños de Pecho de Madrid, 1954. Fuente: Libro conmemorativo del cincuentenario (bodas de oro) de la institución Primer Consultorio de Niños de Pecho, en Madrid (Gota de Leche), 22-1-1904, 22-1-1954, Madrid, 1954. p. 97. 
hospitalización maternal total y que no quede ninguna beneficiaria gestante que verifique su alumbramiento en el ambiente inadecuado de su domicilio" (Asistencia, 1967, p. 8). Para 1968 la Seguridad Social abarcaba al 40-45\% de la población y atendía más de la mitad de todos los partos que se producían en España.

El Seguro de Enfermedad dispuso consultas separadas para niños enfermos (pediatría, tres días a la semana) e higiene de lactantes (puericultura, los tres restantes), hasta que en 1958 se unificaron. Esta situación prefiguró el devenir de la especialidad pediátrica que pasó a obtenerse a través de formación hospitalaria con escasa relación con la higiene infantil.

\section{Transformar lo malo en bueno. Los contenidos del programa de divulgación sanitaria sobre la infancia}

El título de este apartado, tomado de la afirmación programática del médico madrileño de beneficencia Juan Aguirre Barrio, descubre el nervio central del programa de divulgación médica sobre el cuidado de la infancia: "Es de urgente necesidad guiar a los ignorantes, contener a los ... que se equivocan ... transformando paulatinamente lo malo en bueno, lo popular y rutinario en científico y provechoso" (Aguirre, 1885, p. 241).

Las costumbres y creencias de la población, en particular de las clases populares eran 'lo malo', el obstáculo más importante para la salud de los niños (Bernabeu, 2002a, p. 151-3). La mortalidad infantil era debida a "la falta de conocimientos higiénicos y de puericultura de los padres y más principalmente de la mujer, de ausencia de sentido común y gran acopio de preocupaciones supersticiosas y rutinarias" (Sáiz de Llavería, 1914, p. 164). “Los errores y preocupaciones del público en materia de enfermedades cuestan la vida a buen número de niños", aseguraba poco más tarde un médico puericultor (Loste, 1917, p. 32), mientras que otro, tras confesar 40 años de práctica en la atención a los niños, precisaba que: “La muerte de los niños es debida a faltas de sus madres" (G[onzález] Revilla, 1923, p. 69-70). Esta opinión nacía de una visión de género que hacía de las mujeres seres sojuzgados por la biología, custodios exclusivos de sus hijos y de sus hogares por imperativo divino y mentes débiles, proclives al error: “El terreno que mejores condiciones reúne para dar fruto a estas aberraciones es, sin disputa, el cerebro de la mujer, siempre dispuesto a que en él germine lo estupendo, lo maravilloso, lo sobrenatural, los engendros de la ignorancia y del fanatismo, de la presunción necia y de la obcecación indocta" (Salcedo, 1898, p. 15). La ideología sobre la mujer, que trajo la dictadura franquista, reforzó esta visión (Bernabeu, 2002b; Jiménez Lucena, Ruiz Somavilla y Castellanos Guerrero, 2002, p. 208-10) y la mujer equivocada y esquiva para el médico varón 
solo se consideró accesible a través de la labor 'de mujer a mujer' que podía llevar a cabo la divulgadora sanitaria (Lecciones, 1945, p. 16). Contenidos del todo similares, y con la misma periodización, han sido señalados en un estudio reciente acerca de los manuales para las enseñanzas domésticas (de Haro, 1999).

En cualquier caso, desde la perspectiva médica, los peligros partían de la ambivalencia de la consideración de lo popular, al mismo tiempo inocente, por ignorante y contraria, por su radical alteridad. La ignorancia, en sí misma, era inocente, pues procedía de la distancia (natural) que separaba a las clases populares de las elites generadoras de progreso y detentadoras de poder (o, lo que es igual, de la falta de educación). Al mismo tiempo era culpable, en tanto que generadora del 'indeleble estigma' de la terquedad, que provocaba las más feroces resistencias al consejo experto (Salcedo, 1898, p. 4-5 y 14). Lo popular resultaba, así, inaccesible a los consejos y prédicas médicas, mostrándose como el obstáculo más importante para la construcción de un mercado para los médicos de niños, pues las madres recurrían a las " "sabias doctrinas» de las maritornes de enfrente, despreciando los consejos científicos" (Salcedo, 1898, p.628). El mayor riesgo acaecido en el momento del parto, en especial entre la clase pobre, sería el recurso a las "sabias vecinas ... extrañas a la ciencia" (Aguirre, 1885, p. 29). La cercanía cultural, "el mismo grado de ilustración", permitía el ejercicio de aquellas mujeres, llamadas saludadoras, que tenían 'consultas' abiertas para el tratamiento de los niños y que, según las quejas médicas, atendían una gran proporción de los problemas de salud de madres y niños (Perdiguero, 2004, p. 137).

En otras ocasiones se imaginó a la población como una extensión yerma, donde, labrada por los consejos y las recomendaciones médicas, florecerían las semillas de la higiene (Bravo, 1927, p. 21). En este contexto ya no se consideraría a la madre como el enemigo a batir, sino ayudante privilegiado del médico en la atención a los niños y en la extensión de los cuidados higiénicos al conjunto de la población (Tolosa, 1891).

Un elemento delicado en la demarcación de la cultura médica popular, dado el catolicismo agresivo de finales del siglo XIX e inicios del XX, era el posible enfrentamiento con 'la ignorancia religiosa' del pueblo. Es notable el uso frecuente de exvotos y amuletos (por ejemplo, véase Borrás, 1996, p. 46-54) en la salud infantil, de forma particular los dedicados a la dentición. Una alternativa posible era la de trabajar en la separación de los ámbitos espiritual y médico, como proponía Salcedo en línea con su discurso cientifista, resaltando que la doctrina de la Iglesia condenaba las hechicerías y supersticiones (Salcedo, 1898, p. 9-10 y 857). Otra era la opción pragmática de aceptar las costumbres tradicionales, a cambio de introducir nuevas explicaciones significativas. Por ejemplo, 
Benavente, en el contexto de una discusión sobre el mal de ojo, ofrecía a las madres la 'cristianización' de la creencia, de manera que estimulaba a que prendieran 'la cartera con los Santos Evangelios' de la ropita de sus niños para solicitar protección contra enfermedades como el garrotillo o el ataque cerebral, que eran las auténticas amenazas y no 'los ojos de fantásticas hechiceras' (Benavente, 1883b).

La diana de la campaña educadora se fijó en la intimidad familiar y se propuso una completa renovación de los valores vigentes, en sintonía con el programa higienista de moralización más tradicional: confiar y obedecer a la autoridad, incluyendo a la médica; seguir con docilidad y disciplina los consejos expertos. Para hacerlo realidad no se dudó en postular, en ausencia de otros medios, procedimientos de tipo militar: 'el ordeno y mando, tan expeditivo como infalible' (Criado, 1925, p. 131; Bravo, 1927, p. 21). Aunque estos últimos testimonios proceden del tiempo de la primera dictadura militar sufrida por España en el siglo XX, Criado, catedrático de Pediatría de la Universidad de Madrid, ya defendía esa idea doctrinaria de la educación con anterioridad. En 1918, afirmaba en una conferencia que "la esencia de la educación consiste únicamente ... en la subordinación completa de la voluntad del niño a la autoridad de los padres ... A los niños no se les convence, se les manda" (Conferencia, 1918). Y en el supuesto de la educación sanitaria, los profesionales ocupaban el lugar paterno. Bajo el régimen franquista esta visión fue, obviamente, la norma (Bernabeu, 2002b).

El carácter adoctrinador, nada libertario, de esta pedagogía se advierte en la delimitación de la educación popular. En 1883, La madre y el niño, una de las publicaciones dedicadas a popularizar las doctrinas científicas de la crianza, advierte contra la ilustración excesiva de las madres que produciría una 'instrucción funesta, confianza peligrosa' (Pereira, 1883). El objetivo se circunscribe a tener la capacitación precisa para distinguir el momento que se presenta la más leve alteración que exige llamar al médico, un programa mucho más ambicioso que el propuesto por la popularización médica del siglo XVIII. Y puesto que los servicios de beneficencia domiciliaria permitían la asistencia profesional, incluso a los que carecían de medios económicos, la pobreza no debía ser excusa (Salcedo, 1898, p. 604).

El que podemos denominar 'programa máximo' de la medicalización de la infancia implicaría la incorporación de un médico a la familia, puesto que él debía asistir el parto, disponer las reglas higiénicas de crianza, en particular el régimen de alimentación, así como disponer, más adelante, una tabla de ejercicio gimnásticos y, en su caso, de baños de mar (Rojo, 1895, p. 533-4; Sáiz de Llavería, 1914, p. 164-5). Evidentemente, su formulación respondía a un contexto comercial de la práctica médica que se concentraba casi exclusivamente en las capas acomodadas de la sociedad. Un ejemplo lo 
tenemos en la cuestión de las amas de cría. Entre la clase acomoda$\mathrm{da}$, el recurso a la nodriza era un signo de estatus (no en vano era una costumbre acendrada de la Familia Real española), mientras que ese puesto era una vía de sustento para mujeres pobres al servicio de instituciones benéficas como las inclusas. Muchos ayuntamientos (posiblemente la mayoría) en el último decenio del siglo XIX y primeros del siglo XX suministraban pensiones de lactancia a familias pobres.

La preocupación de los médicos respecto a ambos tipos de nodrizas tenía distintos perfiles, pero, en ambos casos, su finalidad era ponerlas bajo vigilancia profesional. Desde finales del siglo XVIII, la literatura médica construyó una imagen absolutamente nega-tiva de aquellas mujeres en tanto que 'receptora[s] de saberes tradicionales erróneos' (Buchan, 1808, p. 357), ignorantes y rudas, adornadas con "todos los errores y preocupaciones del vulgo y no pocos resabios de sus vicios" (Furió, 1881, p. 29). A esta condición básica se achacó la elevada mortandad infantil en las instituciones, por lo que, por extensión - a similitud de lo que ocurría con los conocimientos clínicos, generados en medios institucionales - se aplicaba a los hogares de clase acomodada la conclusión de que su empleo resultaría negativo.

En este punto, no obstante, se topaba con la extensión de la conducta supuestamente errada entre una clientela acomodada. La medicina hubo de reconocer que las amas de cría existían por necesidad ante el 'no escaso número de mujeres' con insuficiente secreción láctea en cantidad o calidad, rechazando no obstante el alejamiento de los recién nacidos de sus hogares familiares (Peiró, 1875; Cirera, 1882, p. 98).

La definición académica de 'la maternidad completa', esto es que la función maternal no acababa con el parto, sino que se extendía, por obligación biológica, moral (cristiana) y patriótica a la lactancia, hizo que el abanico de exenciones técnicamente permisibles se estrechara a lo largo del tiempo, hasta convertirse en excepción: "son contadísimas las mujeres que no pueden lactar" (Vidal, 1903; Sáiz de Llavería, 1914). Sin embargo, las amas sólo fueron definitivamente relegadas con la industrialización higiénica de la leche. Así, en 1893, para Baldomero González Álvarez (1851-1927), con responsabilidades en la inclusa provincial y médico de la Casa Real, el de la nodriza era un mal necesario (1893, p. 30) y a comienzos de siglo los autores catalanes preferían la nodriza al biberón, pese a su confesada admiración por los adelantos en materia de esterilización (Vidal, 1903; Comenge, circa 1906). Las ediciones del Catecismo de Puericultura de Juan Bosch, de 1933 y 1938, defendían la lactancia por nodriza siempre que se alojara en la casa del recién nacido y fuese escrupulosamente seleccionada por consejo médico.

Ahí radicaba el núcleo del argumento, en la introducción del médico dentro del proceso de crianza. Tanto el establecimiento de la indicación como la elección de ama debía ser supervisada por 
profesionales; no existe prácticamente un texto donde no se recoja dicha exigencia. Se dirá: "Sólo el médico está llamado a dictar la medida", y "jamás debe tomarse nodriza sin previo informe facultativo" (Martínez y Suárez, 1887, p. 73 y 76).

En 1877, el gobierno civil de la provincia de Madrid creó una sección de higiene de nodrizas, con la intención de establecer un libro matrícula de nodrizas, a semejanza del servicio de higiene de la prostitución (Castejón, 2001) y un servicio similar se intentó en Barcelona en 1888 (Coll, 1890). También por iniciativa privada, se instalaron centros parecidos de los que en 1886 funcionaban al menos dos en la capital de España, uno de los cuales reconocía y certificaba las condiciones lácteo-sanitarias de unas 600 aspirantes al año (Martínez y Suárez, 1887, p. 76 y 96-107). Una campaña conducida por Manuel Tolosa y la Sociedad Española de Higiene condujo a la promulgación de una ley de protección a la infancia en 1904, con el ambicioso objeto de vigilar a los niños "entregados a lactancia mercenaria, y [a] los que están en casa-cuna, escuela, taller, asilo, etc. y cuanto directa o indirectamente pueda referirse a la vida de los niños durante ese periodo" (art. $1^{\circ}$ ). Si bien la repercusión sanitaria de estos preceptos fue escasa (Perdiguero y Robles, 2004).

\section{Conclusión}

En el desarrollo de una medicina especializada para la infancia tuvo un papel fundamental la realización de una campaña amplia y sostenida de persuasión social que sirvió para acercar a la población y hacer familiares sus ideas y sus prácticas profesionales. En este proceso, extendido entre los siglos XIX y XX, jugó un papel destacado la oferta de bienes y servicios, como los de las gotas de leche, consultorios de lactantes, la obra maternal e infantil del Seguro de Maternidad, así como el Seguro de Enfermedad, a través de los cuales se facilitaba la situación laboral extra-doméstica de las mujeres. Sin embargo, la ideología activa en el seno de esta campaña tuvo un abierto sesgo de género, en la medida en que se defendió una imagen pasiva, nociva y subordinada de las mujeres, en tanto que madres. La principal arma retórica de la campaña fue la contraposición entre la maldad e ignorancia - asignada por entero, y en exclusiva, a los saberes y prácticas populares, incluida la actividad de los sanadores tradicionales $-\mathrm{y}$ la bondad y verdad exclusiva de las propuestas científicas. Esta actitud servía para encubrir los cambios y los fracasos de la medicina científica y la procedencia ilustrada de muchas de las ideas y costumbres criticadas como anticientíficas, a la vez que cerraba los ojos ante las componendas en que incurrían los médicos en la persecución de sus objetivos profesionales por encima de sus propios razonamientos higiénicos, 
como en el caso de las amas de cría. En este sentido, resulta significativo que la campaña de medicalización se apoyara formalmente en una oferta de servicios preventivos, puericultores, de amplio contenido educativo, mientras que, a partir de 1958, los servicios médicos del Seguro de Enfermedad se olvidaron de ello para concentrarse en diagnósticos y tratamientos y la Puericultura pasó, de ser la primera especialidad médica organizada en España, a un título para auxiliares de la más baja escala laboral sanitaria.

\section{BIBLIOGRAFÍA}

Aguirre y Barrio, Juan 1885

Apple, Rima

1995

Apple, Rima

1980

Arteche Aramburu, Ignacio

1928

Asistencia sanitaria de la Seguridad Social en España, (La)

1967

Ballester, Rosa;

Balaguer, Emilio

1995

Benavente, Mariano 1883a

Benavente, Mariano $1883 \mathrm{~b}$

Bernabeu Mestre, Josep $2002 a$

Bernabeu Mestre, Josep

$2002 b$

Bernabeu Mestre, Josep 1995

Bernabeu Mestre, Josep; Perdiguero Gil, Enrique
Mortalidad en la primera infancia, sus causas y medios de atenuarlas.

Madrid: Tip. Hispano-Americana.

Constructing mothers: Scientific motherhood in the nineteenth and twentieth centuries. Social History of Medicine, v. 8, n. 2, p. 161-78.

To be used only under the direction of a physician. Commercial infant feeding and medical practice 1870-1940. Bulletin of the History of Medicine, v. 54 , n. 3 , p. $402-17$.

Consejos a las madres.

El arte de criar a los niños.

Zarauz: Ed. Vasca.

Madrid: Ministerio de Trabajo.

La infancia como valor y como problema en las luchas sanitarias de principios de siglo en España. Dynamis, v. 15, p. 177-92.

Errores populares.

Teta y gloria. La madre y el niño, v. 1, p. 2-4.

Errores populares. Mal de ojo.

La madre y el niño, v. 1, p. 35-7; p. 69-70.

Cultura médica popular y evolución de la mortalidad: los cuidados de salud en la infancia durante la España contemporánea.

Revista de Demografía Histórica, v. 20, n. 2, p. 147-62.

Madres y enfermeras. Demografía y salud en la política poblacionista del primer franquismo, 1939-1950. Revista de Demografía Histórica, v. 20 , n. 1 , p. 123-43.

Malaltia, cultura i població: factors culturals en l'anàlisi demográfica i epidemiológica. In: Barona, Josep L. (ed.) Malaltia i Cultura. València: Seminari d'Estudis sobre la Ciència. p. 153-63.

At the service of Spain and Spanish children: mother-and-child healthcare in Spain during the first two decades of Franco's regime (1939-1963). In: Löwy, Ilana; Krige, John (ed.) Images of disease. Science, public policy and health in post-war Europe.

Luxembourg: European Communities. p. 167-86. 
Borobio Díaz, Patricio 1893

Borrás Llop, José María (dir.) 1996

Bravo Frías, Julio 1927

Buchan, Guillermo 1808

Canoura Balado, Ramón

1925

Carasa, Pedro 1997

Castejón Bolea, Ramón 2001

Cirera Sampere, Juan 1882

Coll y Bofill, Juan 1890

Comenge, Luis 1899

Comenge y Ferrer, Luis c.1906

Criado Aguilar, Francisco 1925

Criado y Aguilar, Francisco 1918

de Haro Oriola, Isabel María 1999

Furió y Roldán, Manuel 1881

Gallego Méndez, $M^{a}$ Teresa

1983

Gómez Redondo, Rosa 1992

Gómez Salvo, Vicente; Camón Gironza, Ramón 1936

González Álvarez, Baldomero 1893
La mortalidad de los niños en Zaragoza.

Sus causas y sus remedios. Zaragoza: C. Ariño.

Historia de la infancia en la España contemporánea, 1834-1936.

Madrid: Ministerio de Trabajo y Asuntos Sociales; Fundación G. Sánchez Ruipérez.

Mortalidad infantil en Madrid y medios para aminorarla.

Madrid: Imp. Municipal.

El conservador de la salud de mujeres y niños.

Madrid: Imp. F. Villalpando.

Principales preceptos higiénicos aplicables a la primera infancia.

Zaragoza: Talleres editoriales del Heraldo de Aragón.

La revolución nacional-asistencial durante el primer franquismo (1936-1940). Historia Contemporánea, n. 16, p. 89-140.

Doctors, social medicine and VD in late nineteenth and early twentiethcentury Spain. In: Davidson, Roger; Hall, Lesley (ed.) Sex, sin and suffering. Venereal disease and European society. London: Routledge. p. 61-75.

Guía de las familias o sea compendio de preceptos higiénicos en relación a la mujer y al niño. Barcelona: Est. Tip. Sucesores N. Ramírez y Cía.

Instituciones creadas en diversos países a favor de la infancia. Dispensarios para niños enfermos. Conferencia pública dada en el Ateneo Barcelonés en la noche del 30 de noviembre de 1889. Barcelona: Imp. Barcelonesa.

Estudios demográficos de Barcelona. Mortalidad infantil, 1889-93; 1894-98. Gaceta médica catalana, v. 22 y 24, passim.

Generación y crianza o higiene de la familia.

Barcelona: José Espasa editor.

La mortalidad en España. Discurso leído en la sesión inaugural del curso 1925-26 en la Real Academia Nacional de Medicina.

Madrid: Imp. J. Cosano.

Conferencia del Dr. Francisco Criado y Aguilar.

Pro-infantia, v. 15, p. 303.

La educación de las mujeres como promotoras de salud en la España contemporánea. Cambios y pervivencias. Tesis doctoral, programa "Estudios de las Mujeres", Dpto. Historia Contemporánea, Universidad de Granada.

[La lactancia mercenaria] Discurso leído en sesión pública celebrada el día 11 de septiembre de 1881 para conmemorar el primer aniversario de la creación de la Academia Médico-Farmacéutica de Albacete. Albacete: Imp. Provincial, p. 21-36.

Mujer, falange y franquismo.

Madrid: Taurus.

La mortalidad infantil española en el siglo XX. Madrid: CIS-Siglo XXI.

Los médicos ante el Seguro de Enfermedad. Enseñanzas que el de Maternidad nos ha proporcionado en la Caja de Aragón, 1931-36.

Zaragoza: Heraldo de Aragón.

Higiene de la alimentación de los niños desde el nacimiento hasta la segunda infancia. Lactancia y destete. Madrid: Carrión Hermanos, impresores. 
González Revilla, Gerardo 1923

Granjel, Luis S. 1965

Huergo, Faustino 1885

Jiménez Lucena, Isabel;

Ruiz Somavilla, Ma José; Castellanos Guerrero, Jesús 2002

Juarros, César 1919

Klaus, Alisa 1993

La Berge, Anne F. 1991

Lecciones de

Puericultura e higiene para cursos de divulgadoras sanitario-rurales. 1945

López Cano, J. 1967

Loste Echeto, Luis 1917

Lozano y Ponce de León, Pablo 1883

Martínez y Suárez, Fermín 1887

Martínez Hernández, Ángel; Comelles, Josep $M^{\underline{a}}$ 1994

Mata Lara, Ana María 1994

Orduña Prada, Mónica 1996

Pascua, Marcelino 1934

Peiró y Serrano, Francisco de Paula 1875
Escuela de puericultura (cuidado de los niños) y de educación sexual. Bilbao: Imp. Echeguren y Zulaica.

Historia de la pediatría española.

Salamanca: Imp. Cervantes.

Cartilla de higiene de los niños para uso de las madres que crían a sus hijos. 3. ed. Oviedo: Impr. Flórez Pintado y Cía.

Un discurso sanitario para un proyecto político. La educación sanitaria en los medios de comunicación de masas durante el primer franquismo. Asclepio, v. 44, n. 1, p. 201-18.

La crianza del hijo.

Madrid: Mundo Latino.

Every child a lion: the origins of maternal and infant health policy in the United States and France, 1890-1920. Ithaca: Cornell University Press.

Mothers and infants, nurses and nursing. Alfred Donné and the medicalization of child care in nineteenth century France. Journal of the History of Medicine and Allied Sciences, v. 46, n. 1, p. 20-43.

Madrid: Sección Femenina de F.E.T. y J.O.N.S.

La Obra Nacional de Auxilio Social. Evolución, situación y perspectivas. In: Problemas fundamentales de beneficencia y asistencia social. Madrid: Ministerio de Gobernación. p. 91-114.

La mortalidad infantil. Causas que determinan su excesiva proporción. Medios de disminuirla. Madrid: Impr. San Roque.

Higiene de los niños y su educación consagrada especialmente a las madres. Madrid: Imp. Enrique Rubiños.

La lactancia bajo todas sus manifestaciones.

Madrid: R. Velasco, impresor.

La medicina popular. ¿Los límites culturales del modelo médico? Revista de Dialectología y Tradiciones Populares, v. 64, n. 2, p. 109-33.

Control social y vida cotidiana de la mujer en la España de Franco. In: Ramos Palomo, Dolores (coord.) Femenino plural. Palabra y memoria de mujeres. Málaga: Universidad de Málaga. p. 221-32.

El Auxilio Social (1936-1940).

La etapa fundacional y los primeros años. Madrid: Escuela Libre.

La mortalidad infantil en España.

Madrid: Dirección General de Sanidad.

Ventajas de la lactancia materna. In: Discursos pronunciados en la inauguración de las sesiones de la Academia de Medicina de Valencia en el año 1875. Valencia: Imp. Ferrer de Orga. p. 19-34. 
Perdiguero, Enrique 2004

Perdiguero, Enrique 1995

Perdiguero, Enrique 1993

Perdiguero Gil, Enrique; Ballester Añón, Rosa 2003

Perdiguero, Enrique;

Bernabeu, Josep 1999

Perdiguero Gil, Enrique; Bernabeu Mestre, Josep

1997

Perdiguero, Enrique;

Bernabeu, Josep 1995

Perdiguero, Enrique; Robles González, Elena 2004

Pereira Pull, F. 1883

Rodríguez Ocaña, Esteban 2006

Rodríguez Ocaña, Esteban

2001

Rodríguez Ocaña, Esteban

1999

Rodríguez Ocaña, Esteban

1996

Rodríguez Ocaña, Esteban

1987

Rodríguez Ocaña, Esteban; Ortiz, Teresa; García-Duarte, Olga 1985
Magical healing in Spain (1875-1936): medical pluralism and the search for hegemony. In: Blécourt, Willem de; Davies, Owen (ed.) Witchcraft continued. Popular magic in modern Europe.

Manchester: Manchester University Press. p. 133-50.

Popularización de la higiene en los manuales de economía doméstica en el tránsito de los siglos XIX al XX. In: Barona, Josep L. (ed.) Malaltia $i$ Cultura. València: Seminari d'Estudis sobre la Ciència. p. 225-50.

La popularització dels coneixements científico-mèdics a la Restauració: el cas de la premsa periòdica d'Alacant. In: Navarro, Víctor et al. (ed.) II Trobades d'Història de la Ciència $i$ de la Tècnica.

Barcelona: Institut d'Estudis Catalans. p. 171-80.

Federico Rubio y el folclore médico. In: Carrillo, Juan L. (ed.) Medicina y sociedad en la España de la segunda mitad del siglo XIX: una aproximación a la obra de Federico Rubio y Galí (1827-1902).

El Puerto de Santa María, Ayuntamiento. p. 475-97.

La Gota de Leche de Alicante (1925-1940). In: Beneito Lloris, Angel et al. (ed.) Beneficència $i$ Sanitat en els municipis valencians (1813-1942).

Alcoi: Seminari d'Estudis sobre la Ciència. p. 291-310.

Burlarse de lo cómico nacido de la tontería humana. El papel otorgado a la población por la divulgación higiénico-sanitaria durante la

Restauración. In: Montiel, Luis; Porras, Isabel (coord.) De la responsabilidad individual a la culpabilización de la víctima. Madrid: Ed. Complutense. p. 55-66.

Morir de dentición. ¿Una creencia popular? In: Arquiola, Elvira; MartínezPérez, José (coord.) Ciencia en expansión: Estudio sobre la difusión de las ideas científicas y médicas en España (siglos XVIII-XX).

Madrid: Ed. Complutense. p. 469-87.

La protección a la infancia y la Sociedad Española de Higiene.

In: Perdiguero Gil, Enrique (comp.) Salvad al niño.

Valencia: Seminari d'Estudis sobre la Ciencia. p. 93-120.

Medicina doméstica.

La madre y el niño, v. 1, p. 53-4.

Medicine as a social political science.

The case of Spain c.1920. Hygiea Internationalis. (En prensa.)

Comodité, ornementation, hygiène. Modernisation urbaine et hygiénisme à l'Espagne du 19ème siècle. In: Bourdelais, Patrice (dir.) Les Hygiénistes: enjeux, modèls et pratiques. Paris: Belin. p. 297-318.

La construcción de la salud infantil. Ciencia, medicina y educación en la transición sanitaria en España. Historia contemporánea, n. 18, p. 19-52.

Una medicina para la infancia. In: Borrás Llop, José $\mathrm{M}^{\mathbf{a}}$ (dir.)

Historia de la infancia en la España contemporánea, 1834-1936. Madrid:

Ministerio Trabajo y Asuntos Sociales/Fundación G. Sánchez Ruipérez. p. $149-83$ y p. $189-92$.

La constitución de la medicina social como disciplina en España, 1884-1923.

Madrid: Ministerio de Sanidad y Consumo.

Los consultorios de lactantes y gotas de leche en España.

Jano, v. 29, n. 663-H, p. 1066-72. 
Rojo Prieto, C. 1895

Rollet-Echalier, Catherine 1990

Sáiz de Llavería, Trinidad 1914

Salcedo Ginestal, Enrique 1898

Salillas Panzano, Rafael 1905

Sánchez López, R. 1990

Tolosa Latour, Manuel 1903

Tolosa Latour, Manuel 1891

Ulecia y Cardona, 1904

Ulecia y Cardona, Rafael 1903

Valera Jiménez, Tomás 1891

Vidal Solares, Francisco 1916

Vidal Solares, Francisco 1915

Wolf, Jacqueline $\mathrm{H}$. 2001
Paralelo entre los niños de las altas esferas sociales y los niños pobres. El Siglo Médico, v. 42, p. 532-5 y 550-3.

La politique à l'égard de la petite enfance sous la IIIème République. Paris: PUF/INED.

La ignorancia de la mujer en los conocimientos de higiene y puericultura como primera causa de la mortalidad infantil.

Gaceta Médica Catalana, v. 37, n. 1, p. 161-8.

Madre e hijo.

Doctrina científica y errores vulgares en obstetricia y ginecología. Madrid: Imp. Ricardo Rojas.

La fascinación en España. Estudio hecho con la información promovida por la Sección de Ciencias Morales y Políticas del Ateneo de Madrid. Madrid: Eduardo Arias.

Mujer española, una sombra de destino en lo universal. Trayectoria histórica de la Sección Femenina de Falange, 1934-1977.

Murcia: Universidad de Murcia.

La protección a la infancia en España.

Leyes y proyectos. Madrid: Est. Tip. Ricardo Fé.

La política doméstica y la higiene. Discurso inaugural leído en la apertura del curso académico de 1891 a 1892. Madrid: Sociedad Española de Higiene.

Arte de criar a los niños (nociones de higiene infantil). Madrid: Administración de la Revista de Medicina y Cirugía Práctica. [1. ed., 1904; 2.0 ed., 1906;

3.0 ed., 1914; 4.0 ed., póstuma, 1919; reimpr. 1924]

Informe acerca de la mortalidad infantil de Madrid, sus principales causas y medios de combatirla, presentado a la Junta Municipal de Sanidad.

Madrid: Impr. Municipal. [El mismo trabajo se presentó al congreso de Deontología Médica, Madrid, 3 a 5 de mayo de 1903 y fue publicado por la Administración de la Revista de Medicina y Cirugía Práctica]

Consejos a las madres.

Conferencia en la Sociedad española de Higiene. Madrid: Lucas Polo.

Consejos a las madres. Aforismos sobre puericultura e higiene de la primera infancia. Barcelona: Tipo-litografía de J. Casamajó. 1903, 1905, 16. ed. (del autor).

Consejos prácticos sobre higiene de la primera infancia, con un apéndice sobre enfermedades de niños. Barcelona: Librería Puig. 1885, 1886; 1887, [Barcelona y Madrid: Librería E. Puig y Admón. Revista Medicina y Cirugía Prácticas. 1891], 1898; Consejos prácticos sobre puericultura e higiene de la infancia, con 170 aforismos. Barcelona: Tipo-litografía de J. Casamajó. 8. ed., 1903;

Puericultura e higiene de la primera infancia.

Barcelona: Luis Gili, librero-editor. 10. ed.

Don't kill your baby. Public health and the decline of breastfeeding in the 19th and 20th centuries. Columbus: Ohio State University Press.

Recibido para publicación en febrero de 2006.

Aprobado para publicación en abril de 2006. 